\title{
Curriculum innovation: The key to recruiting the best and brightest
}

\author{
George J. Magovern, Jr, MD, and Kathleen A. Simpson, BS
}

In this era of change, cardiothoracic surgeons continue to express a high level of satisfaction with the intellectual challenges and rewards of their profession. The interest in pursuing a career in cardiothoracic surgery among American medical students and general surgery residents, however, is unquestionably in decline. Statistics from the Thoracic Surgery Match continue to be concerning. In appointment year 2000, there were 156 total applicants in the Thoracic Surgery Match. ${ }^{1}$ The most recent Match, in June 2010, had only 99 applicants, a decrease of $36 \%{ }^{2}$ Similarly, the number of matched US medical graduates dropped from 96 to 62 during the same period. ${ }^{1,2}$ A recent article in Thoracic Surgery News summarized the results of the June 2010 Match and concluded that these data are likely indicative of a declining pool of well-qualified applicants. $^{2}$ In response, surgeons in the Department of Thoracic and Cardiovascular Surgery at Allegheny General Hospital have developed and initiated an inventive 7-year sequential curriculum that will allow residents to sit board examinations in both vascular and thoracic surgery.

With new technologies evolving, such as the percutaneous aortic valve and less-invasive cardiac assist devices, the need for thoracic surgeons in major metropolitan centers will expand, particularly because the segment of the U.S. population most likely to require cardiovascular care continues to grow, heavily front loaded by the wave of baby boomers now entering the higher-risk age group. ${ }^{3}$ Conservative projections predict a deficit of thoracic surgeons reaching well over $30 \%$ in the next 20 years. ${ }^{4}$

In a recent editorial in The Journal of Thoracic and Cardiovascular Surgery, Kim and colleagues ${ }^{5}$ stated that our specialty must be even more focused and committed to recruitment of the "best and brightest" to cardiothoracic surgery. To accomplish this goal, the Joint Council for Thoracic Surgical Education, headed by Edward Verrier, MD, has undertaken a multifaceted approach to improve cardiothoracic surgery education by developing innovative techniques (including simulation) and redesigning the standard resident training model. Doing this affirms the fact that curriculum

From the Department of Thoracic and Cardiovascular Surgery, Allegheny General Hospital, Pittsburgh, Pa.

Disclosures: Authors have nothing to disclose with regard to commercial support.

Received for publication Nov 17, 2010; revisions received Dec 16, 2010; accepted for publication Jan 3, 2011.

Address for reprints: George J. Magovern, Jr, MD, Allegheny General Hospital, Department of Thoracic and Cardiovascular Surgery, 320 E North Ave, Pittsburgh, PA

15212 (E-mail: gmagover@wpahs.org).

J Thorac Cardiovasc Surg 2011;141:1114-5

$0022-5223 / \$ 36.00$

Copyright (c) 2011 by The American Association for Thoracic Surgery

doi:10.1016/j.jtcvs.2011.01.002 innovation, such as the new 7-year program at Allegheny, is the solution to recruitment of the top medical school graduates into our field. This unique training pathway was specifically developed to answer the concerns voiced by medical students as they consider a career path in surgery.

The critical feature of this new program is the curriculum for postgraduate year 1 , in which the resident works directly with faculty mentors from the Department of Thoracic and Cardiovascular Surgery. The fundamentals of surgery are taught in 4-month blocks supervised by vascular surgery faculty (block 1), general thoracic surgery faculty (block 2 ), and cardiac surgery faculty (block 3 ). A faculty member meets with the first-year resident twice each month for oneon-one medical knowledge review and mentoring. A skills laboratory under the direction of a thoracic surgeon is held bimonthly during years 1 through 3, with additional or remedial training available in subsequent years. An added advantage of this mentored approach is that the resident will be primarily trained by faculty members who are actively involved in and committed to the goal of graduating residents who have achieved board certification in both thoracic and vascular surgery.

Because the curriculum is designed to give the postgraduate year 1 resident exposure to daily clinical decision making, operative, and perioperative skills, and to the professionalism of mature vascular and cardiovascular surgeons, the "delayed gratification" of having to wade through years of general surgery training has been greatly reduced. As a result, the number of applicants to this program has exploded. In the past several years, the applicants to the established 2-year residencies in vascular and thoracic surgery have typically numbered fewer than a dozen. The sequential integrated program has seen more than 70 applications this year.

The advantage of the sequential integrated program in thoracic and cardiovascular surgery is flexibility in training and career pathways. The medical school graduate entering the program is not obligated to finish the sixth and seventh years of formal thoracic surgery training. Although it is expected for students selected for the program to have a genuine and strong interest in both fields, the final decision to finish the thoracic surgery program is not made until the fourth year of the vascular surgery residency. By this time, the resident has had in-depth exposure to and mentoring from all 3 specialties. As a result, an informed and mature decision can be made. The resident can be confident in his or her aptitude for cardiothoracic surgery and understand the scope of the commitment to complete the combined program. 
This curriculum provides not only a robust exposure to training and catheter-based techniques but also to highly complex open procedures on the thoracic and thoracoabdominal aorta. There is concern that the next generations of pure vascular surgeons may not have enough exposure to open vascular procedures as interventional techniques evolve. Such cross-pollination of technologies and educational programs should produce a highly confident, innovative surgeon for the next generation.

Graduates of the combined program will have the flexibility of pursuing a career not only in adult cardiothoracic surgery but also in cardiovascular surgery, thoracic and vascular surgery, pure general thoracic surgery, pure vascular surgery, pure cardiac surgery, or pediatric surgery. This training program will also allow the resident to choose to practice in a cutting-edge, high-powered university hospital setting or in a more conventional community hospital setting, possibly in a rural environment. The university environment would certainly be ideal for the percutaneous valve technology, robotic surgery, and thoracic and peripheral vascular endovascular procedures, as well as for heart and lung transplantation and left ventricular assist device implantation. On the other hand, if the resident so chooses, he or she could practice more traditional general thoracic surgery and cardiac surgery in a large community hospital or possibly practice vascular and thoracic surgery in a smaller more rural setting.

Job opportunities should be more plentiful, as residents will have a more diverse set of operations that they are board certified to perform. Hospitals could elect to hire a single surgeon to perform thoracic and vascular surgery, as opposed to hiring 2 surgeons as is currently the case. In addition, a university practice could hire a cardiovascular surgery specialist who would be current in treating the aorta from the aortic valve to the lower extremities.

The primary disadvantage of this program is that the curriculum is not fully integrated. In the initial 5 years, the resident is in reality a vascular surgery resident with strong exposure to cardiothoracic surgery. He or she answers primarily to the vascular surgery program director, with no formal commitment to thoracic surgery training until the fourth year. Some residents may choose to forego the cardiothoracic surgery training that follows the vascular surgery residency for personal or professional reasons. Because the psychomotor and cognitive competencies necessary for medical students to succeed in cardiothoracic surgery are not yet well understood, the sequential approach of this program certainly allows the resident to decide in time whether the combined career path is the proper choice. In large part, the resident's decision to finish his training in cardiothoracic surgery will be determined by the quality of the exposures and mentoring provided by the cardiothoracic faculty. With a high-quality, engaged faculty, the chances that the resident will finish are strong.

A second disadvantage of a combined program is the fact that it may not be a practical solution in many centers offering separate residency programs in vascular and cardiothoracic surgery. Both of these specialties will need to provide exciting elective rotations to third- and fourth-year medical students to inform the choice of a combined career path. At present, it is certainly not uncommon for program directors in vascular surgery and cardiothoracic surgery to find themselves competing for residents, resources, and clinical material. In this setting, it is unlikely that the 2 programs would see the advantages of a combined, sequential, and partially integrated approach. When viewed as a component of resident education, however, and of the training of cardiovascular surgeons for the next generation, this pathway provides clear benefits to hospitals, patients and the residents who choose this option. Finally, if the sequential integrated program is a success, it could easily lead to a fully integrated 7-year curriculum with a single board exam.

\section{References}

1. Prasad SM, Massad MG, Chedrawy EG, Snow NJ, Yeh JT, Lele H, et al. Weathering the storm: how can thoracic surgery training programs meet the new challenges in the era of less-invasive technologies? J Thorac Cardiovasc Surg. 2009; 137:1317-26.

2. Lesney SB. Making the match in 2010. Thorac Surg News. 2010;6:2.

3. US Census Bureau, Population Division [Internet]. Washington, DC: The Bureau [released 2008 Aug 14; cited 2010 Jul 10]. Table 12. Projections of the population by age and sex for the United States: 2010 to 2050 (NP2008-T12). Available from: http://www.census.gov/population/www/projections/files/nation/summary/ np2008-t12.xls

4. Williams TE Jr, Satiani B, Elison EC. The coming shortage of surgeons. Santa Barbara (CA): ABC-Clio; 2009. p. 72-77.

5. Kim AW, Reddy RM, Higgins RS. Joint Council for Thoracic Surgical Education Subcommittee Best and Brightest. "Back to the future": recruiting the best and brightest into cardiothoracic surgery. J Thorac Cardiovasc Surg. 2010;140: 503-4. 